\title{
In this issue
}

\author{
Fred T. Bosman ${ }^{1}$
}

(C) Springer-Verlag Berlin Heidelberg 2016

In recent years, major advances have been booked in our understanding of the molecular oncogenesis of non-small cell lung cancer (NSCLC), with important implications for final diagnosis and precision treatment, and in this issue, two papers address related new developments in this field. First of all, Bubendorf et al. (DOI 10.1007/s00428-016-2000-3) review rearrangements of the ROS1 gene, which characterize about 1-2 \% of non-small cell lung cancers (NSCLCs). This seems a very minor fraction but given the frequency of lung cancer, it does concern a considerable number of patients per year. In parallel with approval of specific inhibitors of ROS1 kinase activity for treatment of patients with advanced ROS1-positive NSCLC, the need for reliable ROS1 testing has emerged. Tests using a dual color break-apart probe to detect ROS1 rearrangements by fluorescence ín situ hybridization (FISH) have been developed and immunohistochemical detection of expression of ROS1 protein is under way. Molecular tests include dedicated PCR assays, but next-generation sequencing will allow inclusion of ROS1 sequencing in comprehensive testing packages. The authors discuss ROS1 in depth and provide recommendations for

Fred T. Bosman

fred.bosman@citycable.ch

1 Institute of Pathology, University Medical Center (CHUV) of Lausanne, Lausanne, Switzerland

ROS1 testing. The cover image is from this paper and shows ROS1 abnormalities in a FISH test using breakapart probes.

Somewhat in the same vein, Williams et al. (DOI 10.1007/s00428-016-2005-y) address ALK gene rearrangements in NSCLC. The first question their study addresses is the occurrence of ALK rearrangements in long-term ex-smokers, given the fact that as yet, ALK rearrangements have been found predominantly in lifetime non-smokers. In the studied cohort ALK rearrangements were not found in long-term ex-smokers. The second question they ask is whether expression analysis of ALK by immunohistochemistry might replace the currently used FISH test for ALK rearrangement. With the antibody used, high sensitivity was obtained along with acceptable specificity and the authors propose immunohistochemistry as a screening test prior to confirmation by FISH or molecular testing. As ROS1, ALK rearrangement will most likely be included in comprehensive next-generation sequencing test packages.

A further interesting paper in the field of precision oncology is the study by Kim et al. (DOI 10.1007/ s00428-016-2011-0), who examined expression patterns of programmed cell death 1 (PD-1)/PD-1 ligand 1 (PDL1) in extranodal natural killer/T cell lymphoma (ENKTL). ENKTL is associated with Epstein-Barr virus infection, which induces PD-L1 expression. The authors found PD-1+ expression to be rare, but in more than half of the cases PD-L1 was expressed in lymphoma cells as well as in lymphocytes. The most important finding was the correlation of PD-L1 expression with 
better survival, and the authors suggest that PD-L1 expression might be used as a novel prognostic marker.

Even though the diagnosis of cancer tends to be a dominant part of any practicing pathologists' daily life, inflammatory disease including inflammatory bowel disease continues to represent an important part of what they do, both in volume and in complexity. Brown et al. (DOI 10.1007/s00428-0162009-7) address the question how specific morphological signs are in the index biopsy of patients ultimately diagnosed with Crohn's disease. To this end, they compared ileal biopsies from patients diagnosed with 'non-specific ileitis' with tubal gut biopsies (including the upper and lower digestive tract) from patients ultimately diagnosed with Crohn's disease. An important finding was the occurrence of inflammation in several sites of the tubal gut in the latter category, while this was not seen in patients diagnosed with 'non-specific ileitis'. The authors argue that this justifies a full endoscopic examination of the tubal gut, including biopsies, as the presence of multisite inflammation would argue in favor of Crohn's disease. However, a diagnosis of inflammatory bowel disease is never made on biopsies only and the preferred approach towards its initial diagnosis requires close interaction between clinicians and their pathologist(s). 